\title{
Improving accuracy by subpixel smoothing in the finite-difference time domain
}

\author{
A. Farjadpour, David Roundy, Alejandro Rodriguez, M. Ibanescu, Peter Bermel, \\ J. D. Joannopoulos, and Steven G. Johnson \\ Center for Materials Science and Engineering and Research Laboratory of Electronics, Massachusetts Institute of \\ Technology, Cambridge, Massachusetts 02139
}

G. W. Burr

IBM Almaden Research Center, San Jose, California 95120

Received June 16, 2006; accepted July 10, 2006; posted August 3, 2006 (Doc. ID 72037); published September 22, 2006

\begin{abstract}
Finite-difference time-domain (FDTD) methods suffer from reduced accuracy when modeling discontinuous dielectric materials, due to the inhererent discretization (pixelization). We show that accuracy can be significantly improved by using a subpixel smoothing of the dielectric function, but only if the smoothing scheme is properly designed. We develop such a scheme based on a simple criterion taken from perturbation theory and compare it with other published FDTD smoothing methods. In addition to consistently achieving the smallest errors, our scheme is the only one that attains quadratic convergence with resolution for arbitrarily sloped interfaces. Finally, we discuss additional difficulties that arise for sharp dielectric corners. (C) 2006 Optical Society of America

OCIS codes: $000.4430,230.3990,240.6700$.
\end{abstract}

A popular numerical tool for photonics is the finitedifference time-domain (FDTD) method, which discretizes Maxwell's equations on a grid in space and time. ${ }^{1}$ Here, we address difficulties in representing a discontinuous permittivity $(\epsilon)$ on such a grid by proposing an anisotropic subpixel $\epsilon$ smoothing scheme adapted from spectral methods. ${ }^{2-4}$ We show that our method consistently achieves the smallest errors compared with previous smoothing schemes for FDTD. ${ }^{5-7}$ Unlike methods that require modified fieldupdate equations, ${ }^{8}$ our method uses the standard center-difference expressions and is easy to implement (free code is available).

When $\epsilon$ is represented by "pixels" on a grid (or "voxels" in 3D), two difficulties arise. First, a uniform grid makes it more difficult to model small features or to optimize device performance by continuous variation of geometric parameters. Second, the pixelized $\epsilon$ may be a poor representation of the dielectric function: diagonal interfaces produce staircasing, and even interfaces aligned with the grid may be shifted by as much as a pixel. This increases the computational errors and can even degrade the rate of convergence with the grid resolution-as was pointed out in Ref. 8, $\epsilon$ interfaces actually reduce the order of convergence from the nominal quadratic (error $\sim \Delta x^{2}$ ) of standard FDTD to only linear (error $\sim \Delta x$ ). We address both of these difficulties.

The first difficulty is addressed by any smoothing scheme: assign to each pixel some effective $\epsilon$ based on the materials and interfaces in/around the pixel. The effective $\epsilon$ can then vary continuously with geometry. ${ }^{1}$ However, such smoothing perturbs the problem being solved, changing the original discontinuous geometry to a smoothed geometry. Thus, smoothing may actually increase the error. To ensure that the error is reduced, and in fact to restore quadratic convergence, we propose a smoothing with zero first-order effect in Maxwell's equations.

Consider an interface between two isotropic $\epsilon$ materials crossing a pixel, with a unit-normal vector $\mathbf{n}$ perpendicular to the interface (assumed to be locally flat for small pixels, deferring the question of corners until later). We assign to that pixel an inverse dielectric tensor (justified below):

$$
\tilde{\boldsymbol{\epsilon}}^{-1}=\mathbf{P}\left\langle\epsilon^{-1}\right\rangle+(\mathbf{1}-\mathbf{P})\langle\epsilon\rangle^{-1},
$$

where $\mathbf{P}$ is the projection matrix $P_{i j}=n_{i} n_{j}$ onto the normal. The $\langle\cdots\rangle$ denotes an average over the voxel $s \Delta x \times s \Delta y \times s \Delta z$ (in 3D) surrounding the grid point in question, where $s$ is a smoothing diameter in units of the grid spacing ( $s=1$ except where noted). More precisely, FDTD employs a Yee grid in which different field components are computed at different locations, ${ }^{1}$ and we find the averages (1) at each $\mathbf{E}$ component's grid points. This $\tilde{\boldsymbol{\epsilon}}$ is then used to compute $\mathbf{E}=\tilde{\boldsymbol{\epsilon}}^{-1} \mathbf{D}$. For example, to compute $E_{x}$ at its Yee grid point $[i+0.5, j, k]=([i+0.5] \Delta x, j \Delta y, k \Delta z)$, only the first row of the $\tilde{\boldsymbol{\epsilon}}^{-1}$ tensor for that point is needed. When $\tilde{\boldsymbol{\epsilon}}^{-1}$ is not diagonal, we obtain $D_{y}$ and $D_{z}$ at the $E_{x}$ point by simply averaging the $D_{y}$ and $D_{z}$ components from their four adjacent Yee points (similar to Ref. 10). Note that Eq. (1) has the nice property of being Hermitian for real scalar $\epsilon$ and equals $\epsilon^{-1}$ for homogeneous pixels.

Equation (1) corresponds to discretizing a smoothed version of Maxwell's equations, where $\epsilon$ or its inverse has been anistropically convolved with a boxlike smoothing kernel. It was proposed for use with a plane-wave method, ${ }^{2-4}$ based on effectivemedium considerations, but we can also evaluate this 
and other schemes by a simple criterion from perturbation theory. In particular, even before we discretize the problem, smoothing $\epsilon$ causes the solution to be perturbed, and this perturbation can be analyzed via methods recently developed for high-contrast interfaces. ${ }^{11,12}$ To minimize this smoothing error, we simply require that the error be zero to first order in the smoothing diameter $s$ as $s \rightarrow 0$. The remaining smoothing errors will be quadratic in the resolution (except in singular cases as discussed below). Near a flat interface, the effect of a perturbation $\Delta \epsilon$ on computed quantities such as eigenfrequencies ${ }^{11}$ or scattered powers ${ }^{12}$ is proportional to $\Delta \epsilon\left|\mathbf{E}_{\|}\right|^{2}-\Delta\left(\epsilon^{-1}\right)\left|D_{\perp}\right|^{2}$, where $\mathbf{E}_{\|}$and $D$ are the continuous surface-parallel and -perpendicular components of $\mathbf{E}$ and $\mathbf{D}$, respectively. For the first-order change to be zero, $\Delta \epsilon$ must be a tensor, such that $\Delta \epsilon_{\|}$and $\Delta \epsilon_{\perp}^{-1}$ both integrate to zero (e.g., are equal and opposite on the two sides of the interface). A simple choice satisfying this condition is Eq. (1), which uses the mean $\langle\epsilon\rangle$ for the surface-parallel $\mathbf{E}$ components and the harmonic mean $\left\langle\epsilon^{-1}\right\rangle^{-1}$ for the surface-perpendicular component.

Previous smoothing schemes do not satisfy this criterion and are therefore expected to have only linear convergence in general, and they may even have worse errors than unsmoothed FDTD. In particular, we compare our scheme with three other smoothings. The simplest is to use the scalar mean $\langle\epsilon\rangle$ for all components ${ }^{6}$ which is incorrect for the surfacenormal fields. Kaneda et al..$^{5}$ proposed an anistropic smoothing that leads to diagonal $\tilde{\boldsymbol{\epsilon}}^{-1}$ tensors. We also consider the VP-EP scheme, which is exactly the diagonal part of Eq. (1) for $s=1$. Both Kaneda and VP-EP are equivalent to Eq. (1) for flat interfaces oriented along the grid $(x y z)$ directions, but they do not satisfy the perturbation criterion for diagonal interfaces. Yet another method ${ }^{10}$ was found to be numerically unstable for our test cases, which prevented us from evaluating it; however, it is equivalent to Eq. (1) only for flat $x / y / z$ interfaces. Other schemes, not considered here, were developed for perfect conductors $^{1,13}$ or for non-Yee lattices in $2 \mathrm{D} .^{14}$

To evaluate the discretization error, we compute an eigenfrequency $\omega$ of a periodic (square or cubic, period a) lattice of dielectric shapes with 12:1 $\epsilon$ contrast, a photonic crystal. ${ }^{15}$ In particular, we compute the smallest $\omega$ for an arbitrarily chosen Bloch wave vector $\mathbf{k}$ (not aligned with the grid), so that the wavelength is comparable with the feature sizes. We perform a FDTD simulation with Bloch-periodic boundaries and a Gaussian pulse source, analyzing the response with a filter-diagonalization method ${ }^{16}$ to obtain the eigenfrequency $\omega$. This is compared with the exact $\omega_{0}$ from a plane-wave calculation ${ }^{4}$ at a very high resolution, plotting the relative error $\mid \omega$ $-\omega_{0} \mid / \omega_{0}$ versus FDTD resolution. $\omega$ is a good proxy for other common computations, because both the change in the frequency and the scattered power for a small $\Delta \epsilon$ go as $\Delta \epsilon|\mathbf{E}|^{2}$ to lowest order. ${ }^{12}$

Since Kaneda, VP-EP, and our method are equivalent for grid-parallel interfaces (and we obtain qua- dratic convergence for all these methods), we focus instead on a more complicated case: a square lattice of elliptical air holes shown in the inset of Fig. 1 for the TE polarization (E in the 2D plane). Our new method (hollow squares) has the smallest errors by a large margin, while the Kaneda and VP-EP methods are actually worse than no smoothing. As mentioned above, all methods except ours converge linearly, whereas we expect our method to be asymptotically quadratic. As a trick to make the quadratic convergence of our method more apparent, we double the smoothing diameter to $s=2$ (filled squares), at the expense of increasing the absolute error.

The TM polarization (E out of the plane) is not shown, but it is less interesting: all the smoothing methods are equivalent to the simple mean $\epsilon$, all decrease the error compared with no smoothing, and all methods (including no smoothing) exhibit quadratic convergence. Since $\mathbf{E}$ is everywhere continuous, TM is the easy case for numerical computation (and perturbative methods ${ }^{11,12}$ ).

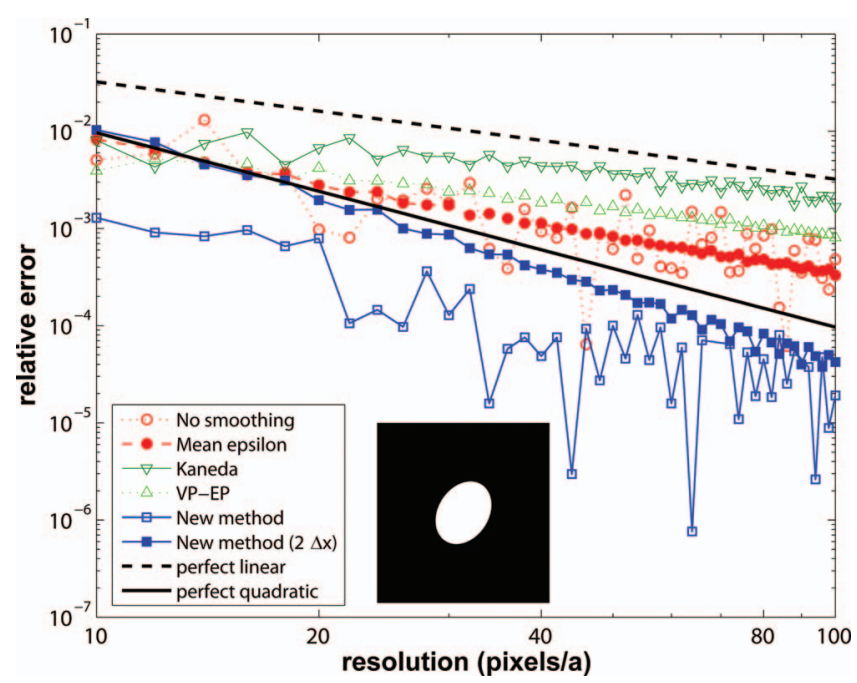

Fig. 1. TE eigenfrequency error versus resolution for a square lattice of elliptical air holes in $\epsilon=12$ (inset).

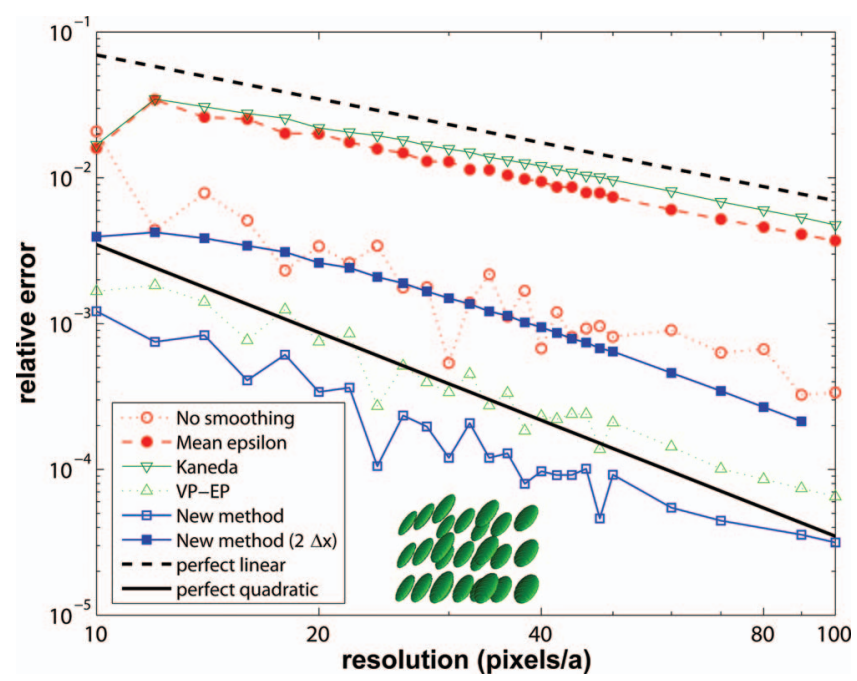

Fig. 2. Eigenfrequency error versus resolution for a cubic lattice of $\epsilon=12$ ellipsoids in air (inset). 


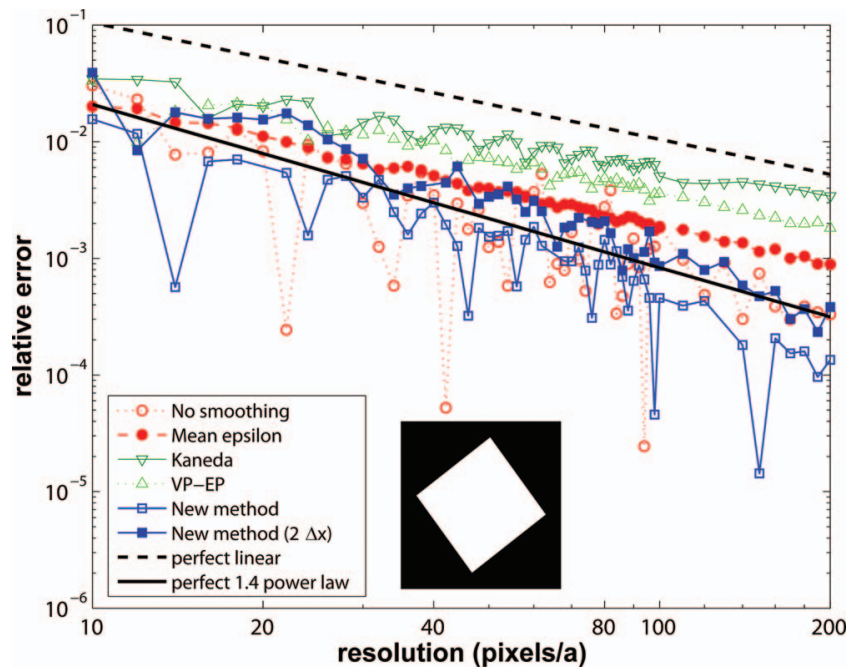

Fig. 3. Degraded accuracy due to field singularities at sharp corners: TE eigenfrequency error versus resolution for a square lattice of tilted-square air holes in $\epsilon=12$ (inset).

In $3 \mathrm{D}$, we used a cubic lattice of $\epsilon=12$ ellipsoids with an arbitrary orientation in air. The results in Fig. 2 again show that the new method has the smallest error and is again quadratic. Notice that the ordering of the other methods has changed, and in general we observe them to yield erratic accuracy.

Finally, we consider a qualitatively different case, in which none of the methods satisfy our zeroperturbation criterion: the presence of a sharp corner leads to a new field singularity. Figure 3 shows the error for a square lattice of tilted air squares in $\epsilon$ $=12$ (inset). Because our new method at least handles the flat edges properly, it still has a lower error than other smoothing schemes, although suboptimal handling of the corner limits the differences. Fits of these data indicate that our method seems to be converging as $\Delta x^{1.4}$, and in fact this can be predicted analytically. Quite generally, any corner leads to a singularity where $\mathbf{E}$ diverges as $r^{p-1}$ for a radius $r$ from the corner, with $p$ given by a transcendental equation in the corner angle and $\epsilon$ values (here, $p \approx 0.702$ ). ${ }^{17}$ This leads to a perturbation in the frequency $\sim \int_{\Delta}|\mathbf{E}|^{2} r \mathrm{~d} r$ $\sim \Delta r^{2 p} \approx \Delta r^{1.404}$, where $\Delta r$ is the size of the perturbation (the pixel). Other smoothing schemes, in contrast, are limited by the linear error from the flat interfaces.

In future work, we hope to extend our method to properly handle corners (where previous work has been limited to right-angle corners ${ }^{18,19}$ ), by using the analytical knowledge of the singularity to design a corner smoothing. Anisotropic $\epsilon$ may be handled similarly to how it was treated in Ref. 3.

This work was supported in part by the Materials Research Science and Engineering Center program of the National Science Foundation under award DMR9400334 and by the Office of Naval Research under award N00014-05-1-0700. A. Farjadpour's e-mail address is ardfar@mit.edu.

\section{References}

1. A. Taflove and S. C. Hagness, Computational Electrodynamics: The Finite-Difference Time-Domain Method, 3rd ed. (Artech, 2005).

2. R. D. Meade, A. M. Rappe, K. D. Brommer, J. D. Joannopoulos, and O. L. Alerhand, Phys. Rev. B 48, 8434 (1993).

3. R. D. Meade, A. M. Rappe, K. D. Brommer, J. D. Joannopoulos, and O. L. Alerhand, Phys. Rev. B 55, 15942 (1997), erratum of Ref. 2.

4. S. G. Johnson and J. D. Joannopoulos, Opt. Express 8, 173 (2001).

5. N. Kaneda, B. Houshmand, and T. Itoh, IEEE Trans. Microwave Theory Tech. 45, 1645 (1997).

6. S. Dey and R. Mittra, IEEE Trans. Microwave Theory Tech. 47, 1737 (1999).

7. A. Mohammadi, H. Nadgaran, and M. Agio, Opt. Express 13, 10367 (2005).

8. A. Ditkowski, K. Dridi, and J. S. Hesthaven, J. Comput. Phys. 170, 39 (2001).

9. "Meep FDTD package," http://jdj.mit.edu/meep.

10. J. Nadobny, D. Sullivan, W. Wlodarczyk, P. Deuflhard, and P. Wust, IEEE Trans. Antennas Propag. 51, 1760 (2003).

11. S. G. Johnson, M. Ibanescu, M. A. Skorobogatiy, O. Weisberg, J. D. Joannopoulos, and Y. Fink, Phys. Rev. E 65, 066611 (2002).

12. S. G. Johnson, M. L. Povinelli, M. Soljačić, A. Karalis, S. Jacobs, and J. D. Joannopoulos, Appl. Phys. B: Photophys. Laser Chem. 81, 283 (2005).

13. I. A. Zagorodnov, R. Schuhmann, and T. Weiland, Int. J. Numer. Model. 16, 127 (2003).

14. S. Moskow, V. Druskin, T. Habashy, P. Lee, and S. Davidycheva, SIAM (Soc. Ind. Appl. Math.) J. Numer. Anal. 36, 442 (1999).

15. J. D. Joannopoulos, R. D. Meade, and J. N. Winn, Photonic Crystals: Molding the Flow of Light (Princeton U. Press, 1995).

16. V. A. Mandelshtam and H. S. Taylor, J. Chem. Phys. 107, 6756 (1997).

17. J. B. Andersen and V. Solodukhov, IEEE Trans. Antennas Propag. 26, 589 (1978).

18. W. W. Lui, C.-L. Xu, W.-P. Huang, K. Yokoyama, and S. Seki, J. Lightwave Technol. 17, 1509 (1999).

19. G. R. Hadley, J. Lightwave Technol. 20, 1219 (2002). 